\begin{tabular}{|c|}
\hline 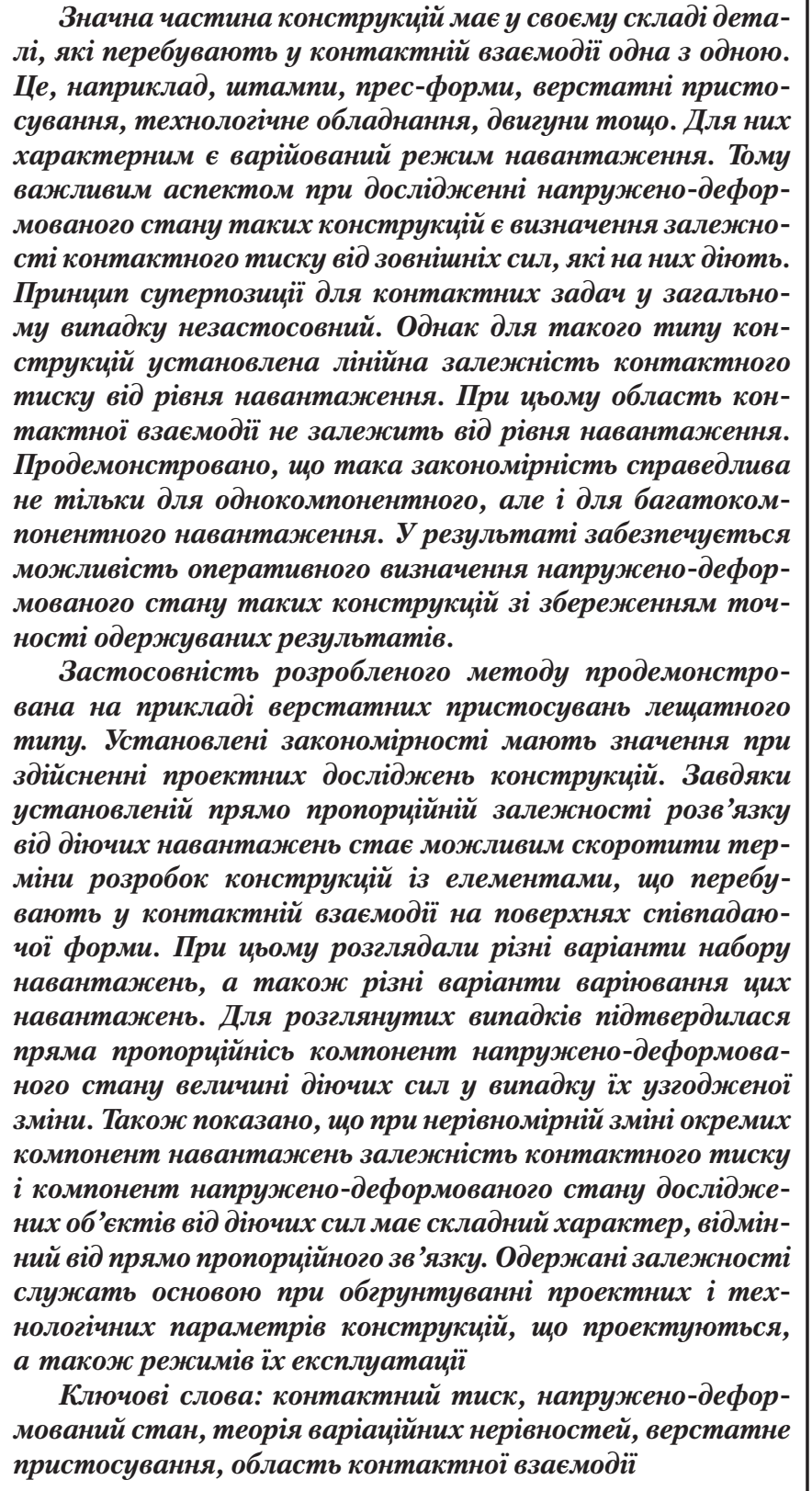 \\
\hline
\end{tabular}

UDC 539.3

DOI: $10.15587 / 1729-4061.2020 .193985$

\section{A SEMI-ANALYTICAL METHOD FOR ANALYS OF CONTACT INTERACTION BETWEEN STRUCTURAL ELEMENTS ALONG ALIGNED SURFACES}

\author{
M. M. Tk ach u k \\ $\mathrm{PhD}$, Senior Researcher* \\ E-mail: m.tkachuk@tmm-sapr.org \\ A. Grabovskiy \\ $\mathrm{PhD}$, Senior Researcher* \\ E-mail: Grabovskiy@tmm-sapr.org \\ M. A. Tk a ch u k \\ Doctor of Technical Sciences, Professor* \\ E-mail: tma@tmm-sapr.org \\ M. S a verska \\ Postgraduate Student* \\ E-mail: m.saverska@tmm-sapr.org \\ I. Hrechka \\ PhD, Associate Professor* \\ E-mail: girinap7@gmail.com \\ *Department of Theory and Computer-Aided \\ Design of Mechanisms and Machines \\ National Technical University \\ «Kharkiv Polytechnic Institute» \\ Kyrpychova str., 2, Kharkiv, Ukraine, 61002
}

\section{Introduction}

A significant share of machine-building structures accounts for such of them whose composition includes parts that are in contact along the aligned (congruent) surfaces. These are, for example, the technological equipment (presses, machine tools, thermoplastic machines), technological equipment (tools, stamps, molds), engines, etc. In this case, it is impossible to avoid solving the problem on the contact interaction between the elements of these structures [1]. The stage of design estimation of the stressed-strained state (SSS) includes, among the variable parameters, not only the structural and technological parameters but the applied current loads as well. Given that in a general case the contact problems are non-linear, this results in that it is necessary to perform additional computer calculations of SSS involving the variation of each component within the system of active loads. Consequently, the volume of computations increases dramatically. That coincides with the design state at which the duration of numerical studies is the most limiting factor. On the other hand, using the simplified analytical dependences to analyze the distribution of the contact pressure and the SSS components is unacceptable in many cases as it results in a significant loss of solution accuracy. Thus, there is a contradiction between the required accuracy and the speed of numerical studies into the SSS of the structures' 
elements. To eliminate this contradiction for a separate class of structures, a new method has been proposed, which makes it possible to combine the advantages of the numerical and analytical modeling of the structures' SSS. This task is relevant because resolving it would provide an opportunity to dramatically improve the efficiency of research into a wide class of structures.

\section{Literature review and problem statement}

A special feature of modern machine building is the wide application and fabrication of progressive structures performing traditional functions. These include, for example, blowers [2,3], pumps [4,6], bearings [5], hydraulic machines [7-11], etc. Papers [2,3] defined the characteristics of these complex structures by using numerical and experimental modeling. However, these papers do not describe studying the SSS of the blowers' individual elements considering their contact interaction. Articles [4,5] describe the numerical and experimental studies into the elements of pumps and roller bearings. However, there are certain concerns regarding the analysis of performance of these elements in terms of their contact interaction. They testify to the insufficient attention to the distributions of SSS components, predetermined by the action of the contact pressure. Papers [7-11] investigated the working processes in pumps, hydraulic motors, and rotors in mechatronic systems. However, not enough attention was paid to the nonlinear processes and states that are implemented in such structures.

Thus, the cited studies substantiate the rational shape, size, and operational modes of such structures through the development and application of high-efficiency models and research methods. In many cases, such studies lead to the need to solve the nonlinear analysis problems [4-6]. However, that significantly increases the volume of numerical studies as it contradicts the principle of superposition, which is inherent in the linear systems. Thus, an option to remove this contradiction is the prospect to establish, at least for particular cases, patterns in the performance of solutions to the nonlinear problems involving variation, specifically, of the applied loads [8-10]. This would create opportunities for combining the benefits of numerical and analytical research methods. On the one hand, this implies a wide scope of application and high accuracy, on the other hand, efficiency, as well as the visibility and the forecasting of trends in the change in analysis results when varying loads.

In particular, it is very important to analyze the contact interaction between bodies along the matching surfaces. It is a rather broad and widespread class of structures. An important aspect for their functioning is to provide the required level of strength or rigidity. And this, in turn, is determined, to a large extent, by the contact interaction within a system of bodies. This has not been a fully investigated field up to now [1].

At present, there are a lot of issues related to studying the contact between elastic bodies. The contact interaction between structural elements makes up an important and extensive field in mechanics [1]. A wide range of methods and models were constructed to solve problems on contact interaction between structural elements. Specifically, papers [1, 12-14]. These papers employ the methods of variational inequalities or a Kalker's variational princi- ple [14-16] to solve the contact problems that occur in this case. These methods and models cover a broad class of contact problems but have a series of drawbacks. Among them, the lack of a universal mechanism for taking into consideration different factors, in particular, the properties of materials in the intermediate layers between the bodies in contact. Studies [17-19] address the models of properties of the rough surfaces of contacting bodies, taking into consideration their wave-like shapes. Papers [20-22] describe the effect of a geometric shape of surfaces and the distribution of a material's properties on the contact interaction between elastic bodies. These models are of great methodological significance. However, there is an unresolved issue regarding the integration of these models into the problems within a particular subject area. Works $[23,24]$ tackle the contact involving adhesion. Papers [25, 26] described a JKR model to analyze the contact between bodies involving with adhesion. These models provide an opportunity to determine the dependence of not only the forces of compression but also the forces of stretching when elastic bodies are in contact. However, these models pose some difficulties for numerical implementation. Articles [27-29] describe the use of a boundary element method for the analysis of contact interaction between elastic bodies. This method has some advantages over the finite element method. However, there are certain limitations when it is applied to actual machine-building structures.

Some factors that are important to analyzing the contact interaction between elastic bodies were taken into consideration in a series of studies. Specifically, this is the impact of bolted joints [30, 31], the presence of a linear or nonlinear intermediate layer between contacting bodies [32-34], etc. Furthermore, several papers [35-37] consider the special properties of thin-walled structures or the reinforced surface layers in machine parts affecting their strength and rigidity. At the same time, classical approaches, described in works [38-40], regarding the models of continuum mechanics, a finite element method and the minimization methods, form the basis only for a general variational statement of the problems on analyzing the contact interaction. However, their direct application does not make it possible to directly state and solve all the types of emerging contact problems.

Thus, current approaches, methods, models, and research tools do not provide for a direct opportunity to analyze patterns in the distribution of the contact pressure between bodies along the aligned surfaces. However, such a possibility is provided by the advancements reported in papers [34, 41, 42]. These findings extend such known methods as a Kalker's variational principle and a finite element method [16, 28]. In this case, it becomes possible to avoid the shortcomings associated with traditional methods and models. At the same time, this provides an opportunity to apply these findings to solve problems on the contact interaction between bodies along the aligned surfaces. This naturally accounts for the properties of intermediate layers.

It is necessary to pay attention to the fact that a series of studies have established the dependences of changes in the solutions to problems on the stressed-strained state of structures' elements at the contact surfaces that match in shape [34, 41, 42]. However, there remains the uncertain performance of the solutions to such problems under a multi-component set of loads, which consists not of one or two but several forces. 


\section{The aim and objectives of the study}

The aim of this study is to construct a semi-analytic method to explore the SSS of structures with elements that are in contact with the aligned (congruent) surfaces. That would make it possible to accelerate the solving of problems on contact interaction between structural elements along the aligned surfaces.

To accomplish the aim, the following tasks have been set:

- to devise models and research methods of the contact interaction between structures with elements that interact along the aligned (congruent) surfaces;

- to solve the test numerical problems;

- to analyze changes in the distribution of the SSS components and the contact pressure in the examined objects.

\section{Models and research methods of the contact interaction between structures with the elements that interact along the aligned (congruent) surfaces}

We shall consider a system of bodies in contact, which occupy volumes $\Omega_{n}, n=1, \ldots, N$ (here, $N$ is the number of bodies) in space. The following components of the SSS of these bodies are determined: a vector of displacements of the bodies' points $\vec{u}^{(n)}$, the deformation $\varepsilon^{(n)}$ and stresses $\sigma^{(n)}$ tensors. These components form the fields, that is, the distributions of these quantities relative to the spatial coordinates $x_{k}$, $k=1,2,3$, which are the components of radius-vector $\vec{r}$ of an arbitrary point $M_{n} \in \Omega_{n}$. The SSS components satisfy the system of equations from the continuum mechanics [38]:

$$
\begin{aligned}
& 2 \varepsilon_{i j}=u_{i, j}+u_{j, i} ; \quad \sigma_{i j, j}+f_{j}=0 ; \\
& \sigma_{i j}=C_{i j k l} \varepsilon_{k l}, \quad i, j, k, l=1,2,3 .
\end{aligned}
$$

Here, the $u, \varepsilon, \sigma$ components are featured without an index $n$ associated with the belonging to a particular body, meaning they hold to all bodies.

In equations (1), $f_{i}$ are the components of volumetric loads, $C_{i j k l}$ are the components of the tensor of elastic constants of a material.

Equations (1) are supplemented with the boundary conditions:

$$
\left.u^{(n)}\right|_{S_{u}^{(n)}}=u^{(n) *},\left.\quad \sigma^{(n)}\right|_{S_{\sigma}^{(n)}}=\sigma^{(n) *},
$$

where $u^{*}, \sigma^{*}$ are the set displacements and loads at surfaces $S_{u}^{(n)}$ and $S_{\sigma}^{(n)}$, respectively.
In addition, on the parts of contact surfaces $S_{c}^{(p q)}$ the following conditions for impenetrability are met:

$$
u_{\mathrm{v}}^{(p)}+u_{\mathrm{v}}^{(q)} \leq \delta_{p q} ; \quad p, q=1, \ldots, N,
$$

where $u_{v}^{(p)}, u_{v}^{(q)}$ are the displacements in the direction of a normal $v$ to surface $S_{c}^{(p q)} ; \delta_{p q}\left(r_{p}\right) \equiv \delta_{p q}\left(r_{q}\right)$ is the distribution of a gap between bodies $\Omega_{p}$ and $\Omega_{q} ; N$ is the number of bodies in contact.

In a general case, $\delta_{p q}\left(r_{p}\right)$ is a certain arbitrary gap distribution. However, for a certain class of structures (Fig. 1), $\delta_{p q} \equiv 0$. If, in addition, $S_{c}$ are the parts of the planes, then, in the absence of friction, we attain the variational statement of the contact problem [12-14]:

$$
I(u) \rightarrow \min ,
$$

where $u=\bigcup u^{(n)}$ are the distributions of displacements of points of the contacting bodies that satisfy conditions (2), (3).

The functionality of complete energy of the examined system of contact bodies [12-14]:

$$
\begin{aligned}
& I(u)=\frac{1}{2} \sum_{n=1}^{N} \int_{\Omega_{n}} \sigma_{i j}^{(n)}\left(u^{(n)}\right) \cdot \varepsilon_{i j}^{(u)}\left(u^{(n)}\right) \mathrm{d} \Omega_{n}- \\
& -\sum \int_{S_{\sigma}^{(n)}} \sigma^{(n)^{*}} \cdot u^{(n)} \mathrm{d} S_{\sigma}^{(n)},
\end{aligned}
$$

was defined on a set of displacements $u$. When sampling this functionality by using a finite element method (FEM) [32], the functional takes the quadratic form $J$ :

$$
J(U)=\frac{1}{2} \sum_{i, j} K_{i j} U_{i} U_{j}-\sum_{i} f_{i} U_{i}
$$

where $U$ is the array of nodal displacements with components $U_{i} ; f$ is the array of nodal forces with components $f_{i}$; $K_{i j}$ are the components of a rigidity matrix $K$.

In this case, ratios (3) take the form:

$$
U_{e}-U_{g} \leq 0
$$

where the $e, g$ indexes correspond to the normal displacements of conjugated nodes in the finite-element models of the bodies in contact.

Thus, the original problem takes the form of a problem on quadratic programming $[40,43,44]$ : find a minimum of function (6) on the set given by conditions (7). Such statements are considered in papers [32, 34, 41, 42]. However, the resolving relationships in these papers are constructed for a general case of the distribution of the initial gap between the bodies in contact.

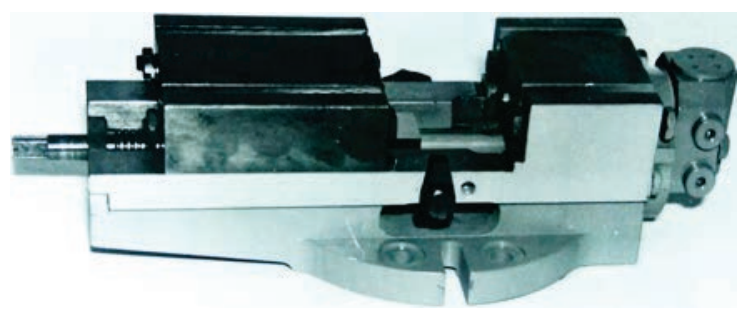

$a$

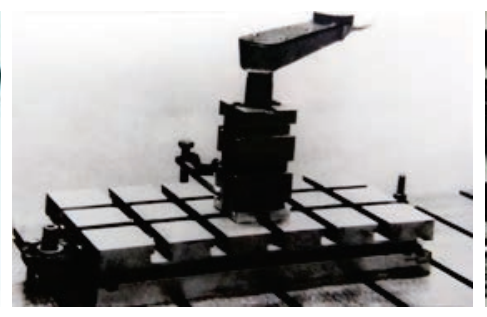

$b$

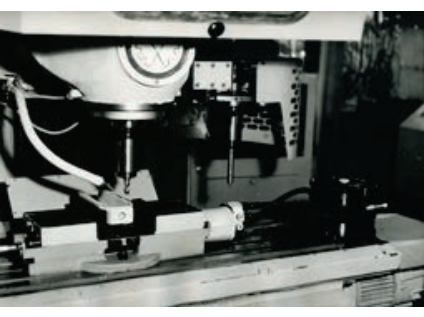

Fig. 1. Examples of structures with the contact of bodies along the aligned surfaces: $a$ - vise with a single movable jaw; $b$ - universal prefabricated assembly; $c$ - vise with two movable jaws 
As noted in studies [41, 42], for the case of stamps and molds, the partial regularities for the distributions of the contact pressure are confirmed. These patterns imply that for the single- and two-component systems of forces one observes a directly proportional dependence between the level of the applied forces and the level of the contact pressure. At the same time, the contact area remains unchanged when the operating forces are varying. There is a task to determine the legitimacy of these features for the case of a multi-component system of forces that act on the examined structures.

For the case of an arbitrary system of forces acting on structures with bodies in contact along the aligned surfaces, it is possible, similarly to [41], to consider a minimum of the quadratic form (6) over regions assigned by constraints (7) [43, 44]. As shown by Fig. 2, in this case, we obtain a proportional dependence of the solution, that is the point of a minimum from the load level. In other words, if one increases all components of the array $f$ of nodal forces by $\tau$ times, the solution will also change proportionally. In this case, if the inequality holds in ratios (7), we obtain an unconditional minimum $J$ (points $O_{0}, O_{\tau}$ in Fig. 2). If the equality holds in (7), then we obtain a nominal minimum (points $T_{0}$, $T_{\tau}$ in Fig. 2). The subsets of nodes, in which the equality and inequality hold, remain unchanged. Physically, this means the constant contact area.

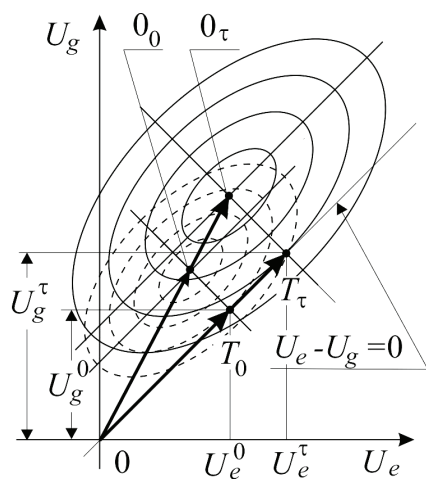

Fig. 2. Lines of the function $J$ levels in cross-section $U_{e}-U_{g}$

Thus, for the discretized model of the examined object, we have shown a known validity [1] of the direct proportional dependence of the SSS components within a system of bodies in contact along the aligned surfaces on the level of the current system of forces. fixing effort $Q$, generated by a force cylinder. The third component is the force of cutting a part $N$, which acts from the side of a tool when performing a technological operation. The contact is assigned between the sole of the vise and the base.
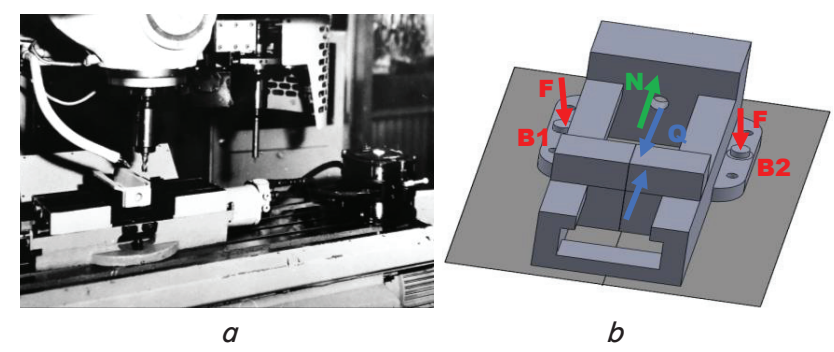

Fig. 3. Machine assemblies of the vise type:

$a$ - image of the examined object; $b$ - estimation model and the scheme of forces application

Let us accept the rated values $F_{0}=30 \mathrm{kN}, Q_{0}=60 \mathrm{kN}$, $N_{0}=40 \mathrm{kN}$. In this case, the current force value is:

$$
F=\tau_{F} \cdot F_{0} ; Q=\tau_{Q} \cdot Q_{0} ; \quad N=\tau_{N} \cdot N_{0} .
$$

The following problems are stated: to vary coefficients $\tau_{F}$, $\tau_{Q}, \tau_{N}$ and to derive dependences:

$$
\begin{aligned}
& \hat{q}=\frac{q_{\max }\left(\tau_{F}, \tau_{Q}, \tau_{N}\right)}{q_{\max }(1,1,1)} ; \quad \sigma^{\wedge}=\frac{\sigma_{\max }\left(\tau_{F}, \tau_{Q}, \tau_{N}\right)}{\sigma_{\max }(1,1,1)} ; \\
& \hat{u}=\frac{u_{\max }\left(\tau_{F}, \tau_{Q}, \tau_{N}\right)}{u_{\max }(1,1,1)}
\end{aligned}
$$

where $q_{\max }$ is the maximum contact pressure; $\sigma_{\max }$ is the maximum equivalent stresses by Mises; $u_{\max }$ is the maximum complete displacements at the specified values $\tau_{F}, \tau_{Q}, \tau_{N}$.

Based on the research results, we construct the dependences of non-dimensional characteristics $q^{\wedge}, \sigma^{\wedge}, u^{\wedge}$ on parameters $\tau_{F}, \tau_{Q}, \tau_{N}$. Define the following scenarios for varying parameters $\tau$ :

1) $\tau_{Q}=\tau_{N}=0 ; \tau_{F} \in[0.1 ; 10]$

2) $\tau_{Q}=0 ; \tau_{N} \in[0.1 ; 10] ; \tau_{F} \in[0.1 ; 10]$;

3) $\tau_{Q} \equiv \tau_{N} \equiv \tau_{F} \equiv \tau \in[0.1 ; 10]$.

The results from solving problems 1-3 are analyzed further:

1. At variable $\tau_{F}\left(\tau_{O}=\tau_{N} \equiv 0\right)$, we obtain, by using the finiteelement packets, an unchanging pattern of the distribution of the contact pressure, stresses, and displacements (Fig. 4-6).

The contact area is constant.

The dependence $q^{\wedge}\left(0,0, \tau_{F}\right), \sigma^{\wedge}\left(0,0, \tau_{F}\right), u^{\wedge}\left(0,0, \tau_{F}\right)$ on $\tau_{F}$ is directly proportional (Fig. 7).

\section{Solving test numerical problems}

We shall consider a machine's assembly of the vise type as an example of the examined structure. It is characterized by the presence of three components of active forces. The first is created by the fixing forces $F$, which operate at bolts B1 and B2 (Fig. 3). The second component is the

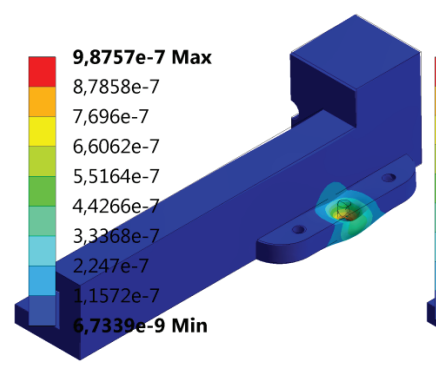

$a$

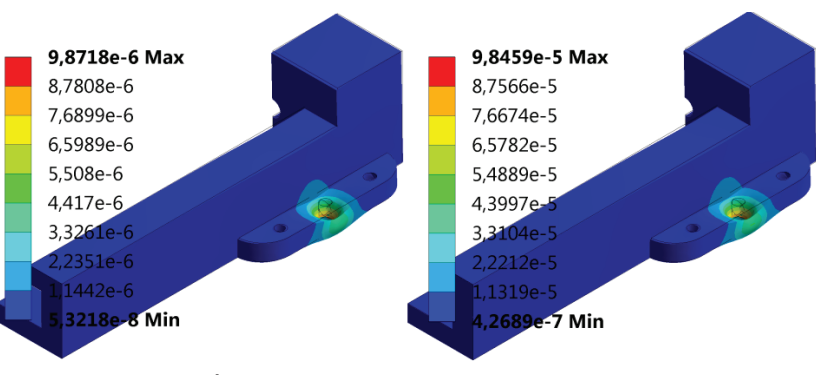

$b$ $c$
Fig. 4. Patterns of displacement distributions for the characteristic values of a bolt fixing force: $a-\tau_{F}=0.1 ; b-\tau_{F}=1 ; c-\tau_{F}=10$ 


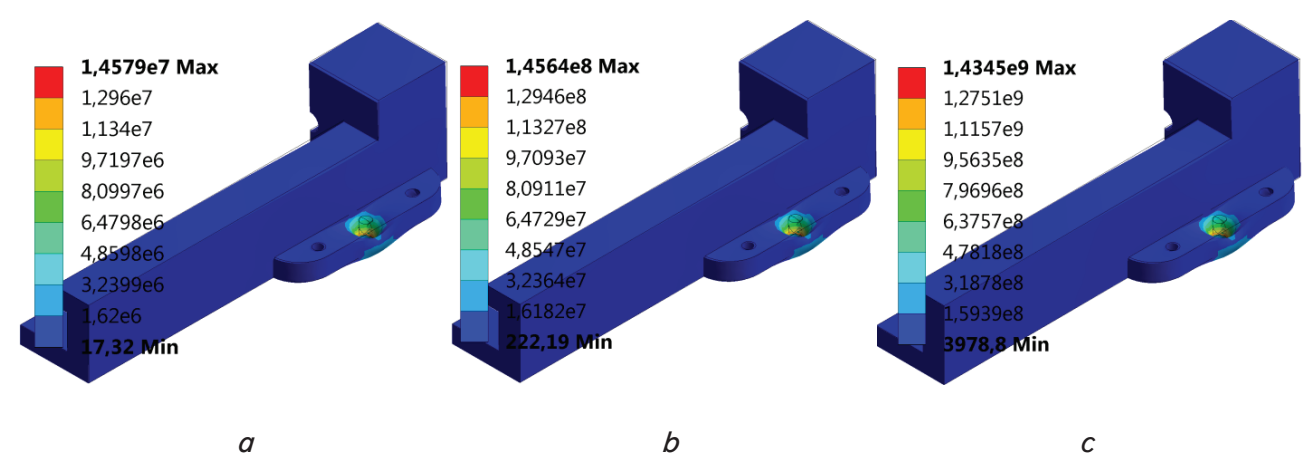

Fig. 5. Patterns of stress distribution for the characteristic values of a bolt fixing force:

$$
a-\tau_{F}=0.1 ; b-\tau_{F}=1 ; c-\tau_{F}=10
$$

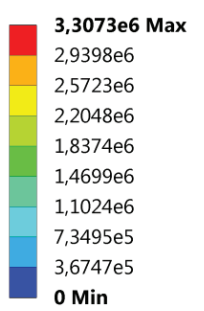

3,3067e7 Max

2,9393e7

$2,5719 \mathrm{e} 7$

$2,2045 \mathrm{e} 7$

$1,837 \mathrm{e} 7$

$1,4696 \mathrm{e} 7$

$1,1022 \mathrm{e} 7$

$7,3482 \mathrm{e} 6$

3,6741e6

0 Min

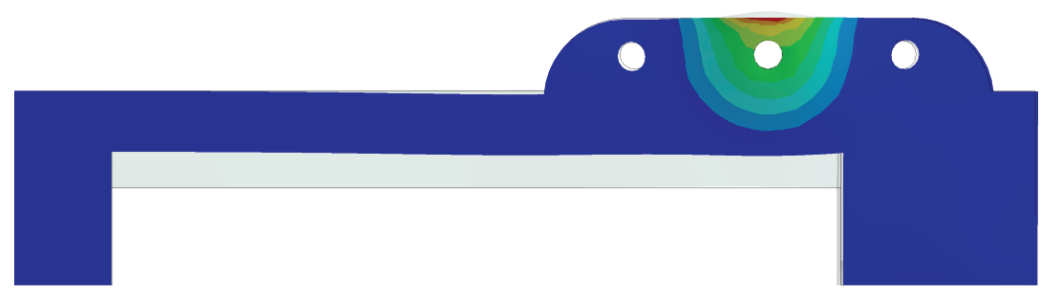

$a$

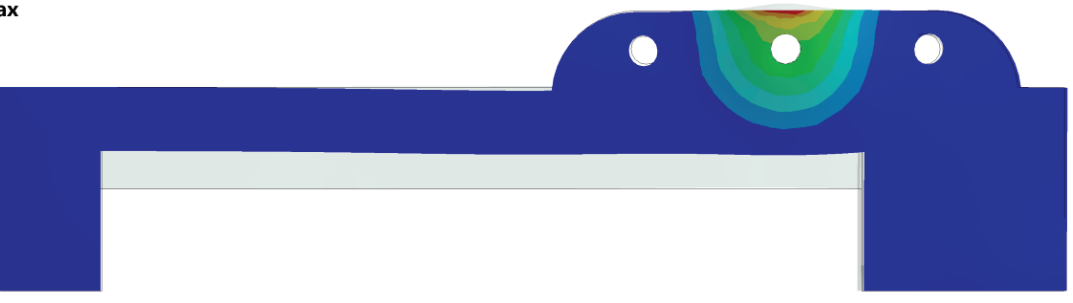

$b$

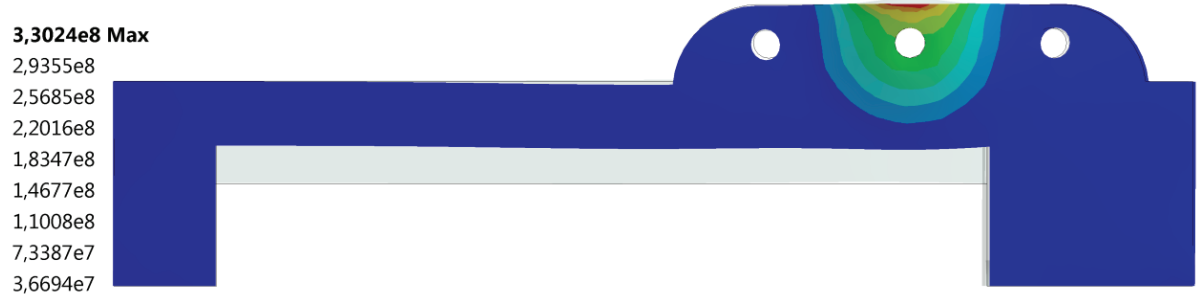

0 Min

C

Fig. 6. Patterns of the contact pressure distribution for the characteristic values of a bolt fixing force:

$$
a-\tau_{F}=0.1 ; b-\tau_{F}=1 ; c-\tau_{F}=10
$$

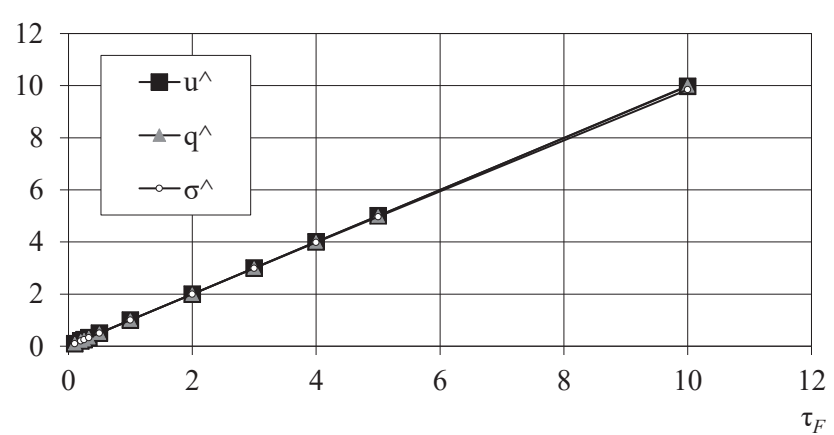

Fig. 7. Dependence of change in the non-dimensional characteristics $\hat{q}, \hat{\sigma}, \hat{u}$ on the degree of variation of a bolt fixing force $\tau_{F}$
2. At variable $\tau_{F}, \tau_{Q}\left(\tau_{N}=0\right)$, the pattern of the distribution of the contact pressure, stresses, and displacements is heavily dependent on the $\tau_{F}, \tau_{Q}$ ratio (Fig. 8-10).

The contact area is variable.

The dependence $q^{\wedge}\left(0, \tau_{F}, \tau_{Q}\right)$ is complex in nature (Fig. 11).

At the same time, at the fixed ratios of $\tau_{N}, \tau_{F}$, there is a directly proportional relationship of the $q^{\wedge}, \sigma^{\wedge}, u^{\wedge}$ characteristics on $\tau=\tau_{N}=\tau_{F}$.

3. Under the aligned variation $\tau_{Q}=\tau_{N}=\tau_{F}=\tau$, there is a linear pattern of the contact pressure, stresses, and displacements distributions when changing $\tau$ (Fig. 12-14).

The $q^{\wedge}, \sigma^{\wedge}, u^{\wedge}$ quantities are directly proportional to $\tau$ (Fig. 15).

The contact area is unchanged. 


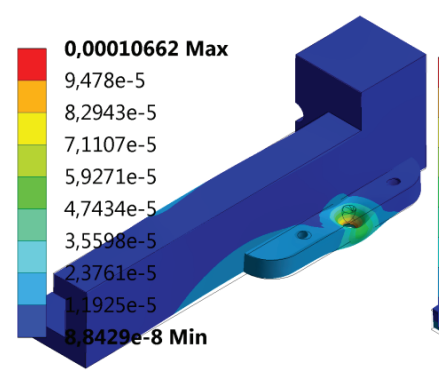

$a$

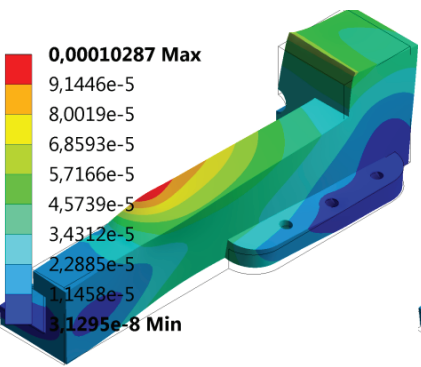

$b$

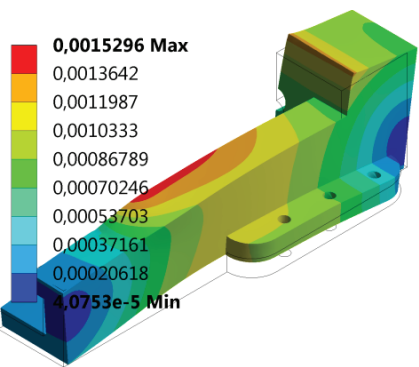

$c$

Fig. 8. Patterns of the displacement distributions for different variation options $\tau_{N}, \tau_{Q}$ : $a-\tau_{F}=10, \tau_{Q}=0.1 ; b-\tau_{F}=1, \tau_{Q}=1 ; c-\tau_{F}=0.1, \tau_{Q}=10$

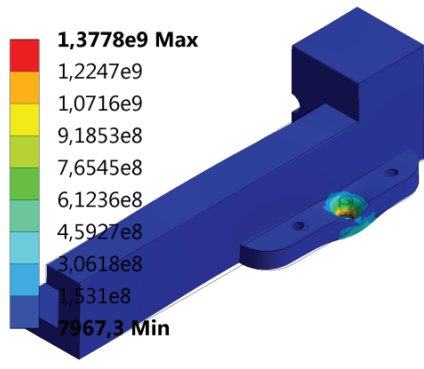

$a$

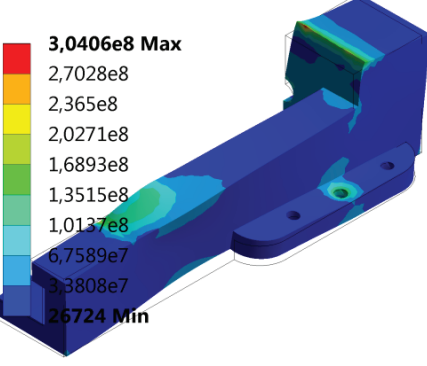

$b$

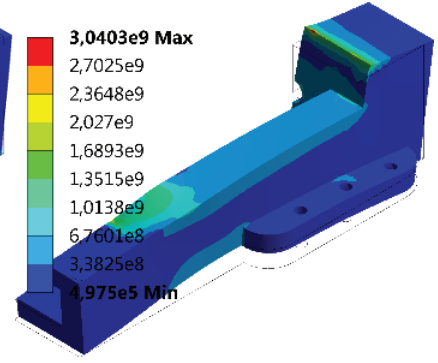

C

Fig. 9. Patterns of the stress distribution for different variation options $\tau_{N}, \tau_{F}$ : $a-\tau_{F}=10, \tau_{Q}=0.1 ; b-\tau_{F}=1, \tau_{Q}=1 ; c-\tau_{F}=0.1, \tau_{Q}=10$

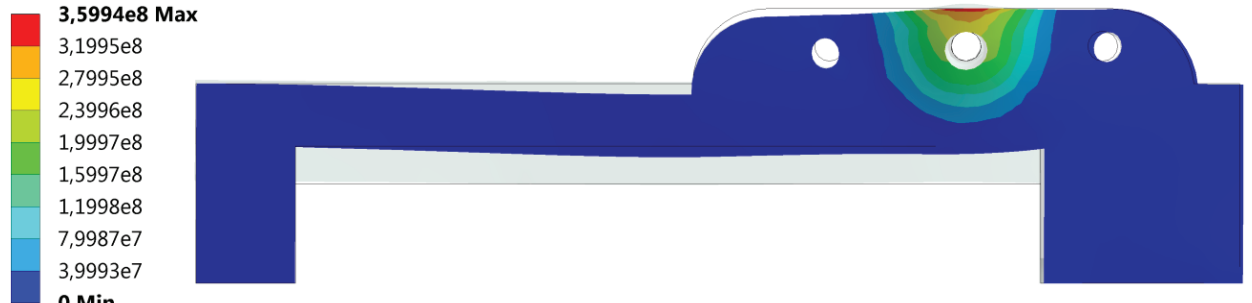

0 Min
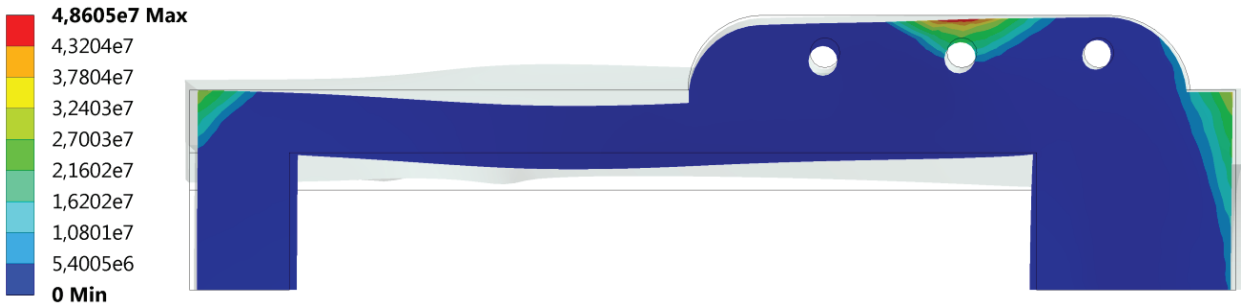

$b$

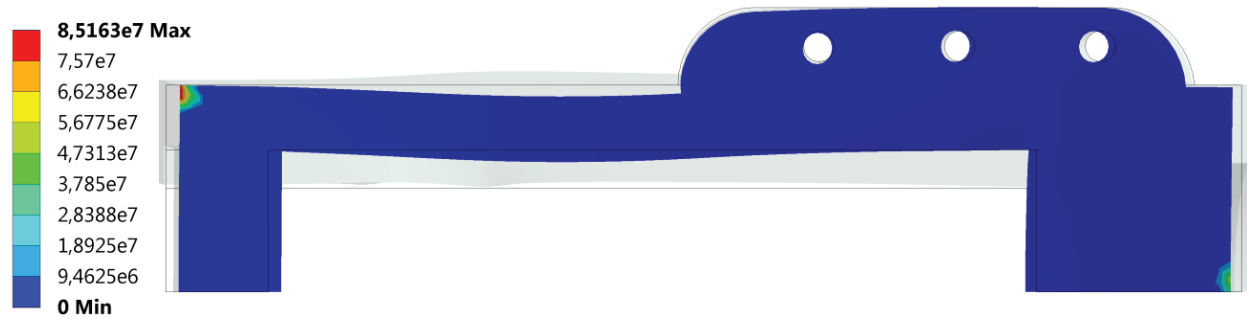

$c$

Fig. 10. Patterns of the contact pressure distribution for different variation options: $a-\tau_{F}=10, \tau_{Q}=0.1 ; b-\tau_{F}=1, \tau_{Q}=1 ; c-\tau_{F}=0.1, \tau_{Q}=10$ 


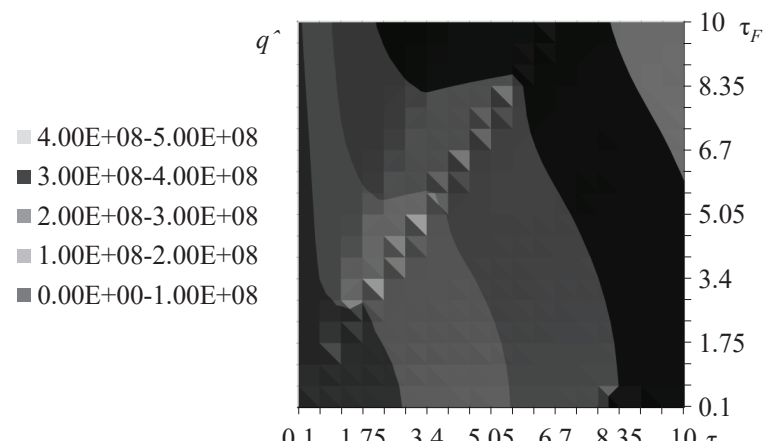

$\begin{array}{lllllll}0.1 & 1.75 & 3.4 & 5.05 & 6.7 & 8.35 & 10 \tau_{Q}\end{array}$

Fig. 11. Dependence $q^{\wedge}$ on $\tau_{Q}, \tau_{F}$
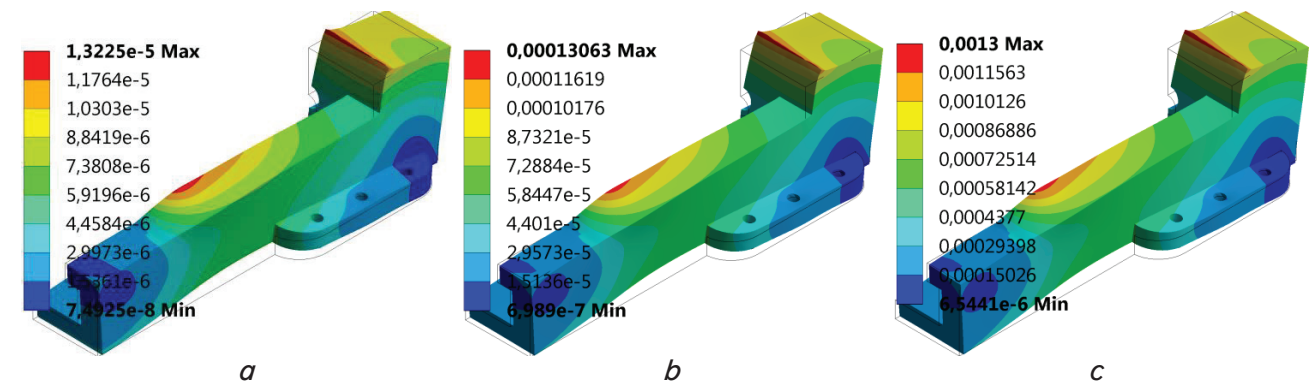

Fig. 12. Patterns of the displacement distributions under aligned variation at $\tau_{Q}=\tau_{N}=\tau_{F}=\tau: a-\tau=0.1 ; b-\tau=1 ; c-\tau=10$
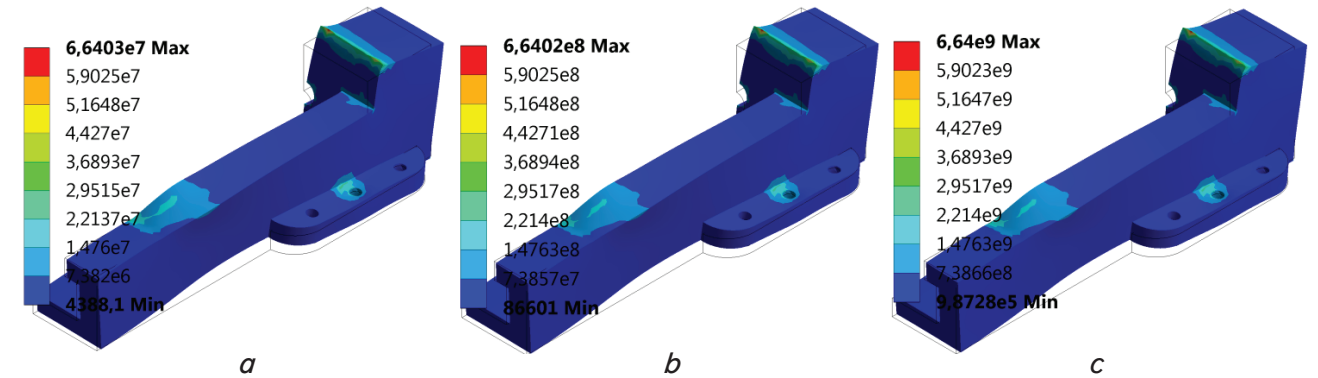

Fig. 13. Patterns of the stress distributions under aligned variation at $\tau_{Q}=\tau_{N}=\tau_{F}=\tau: a-\tau=0.1 ; b-\tau=1 ; c-\tau=10$

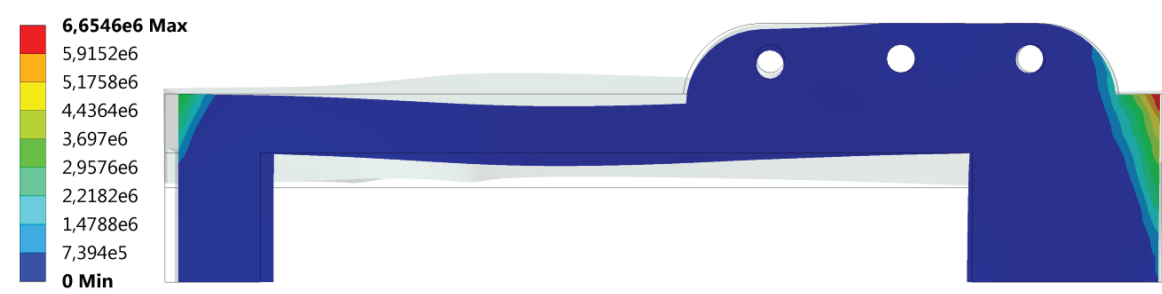

$a$
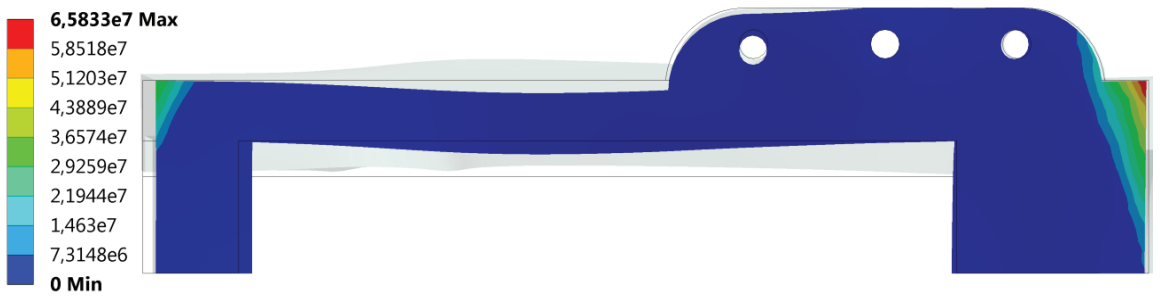

$b$

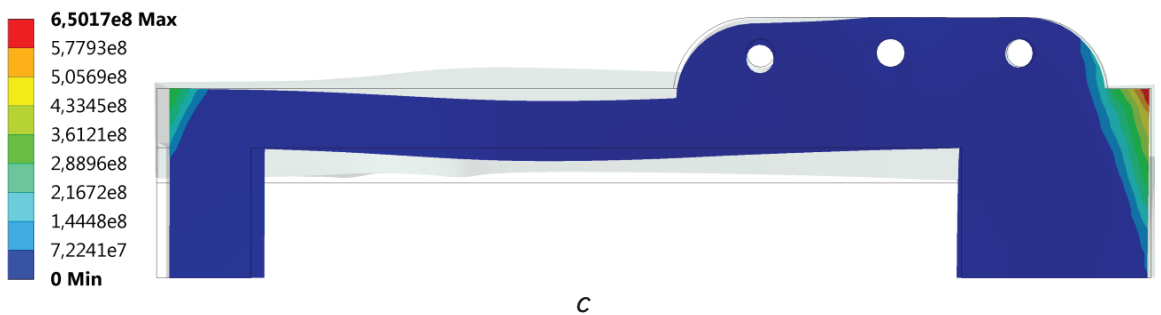

Fig. 14. Patterns of the contact pressure distribution under aligned variation at $\tau_{Q}=\tau_{N}=\tau_{F}=\tau: a-\tau=0.1 ; b-\tau=1 ; c-\tau=10$ 


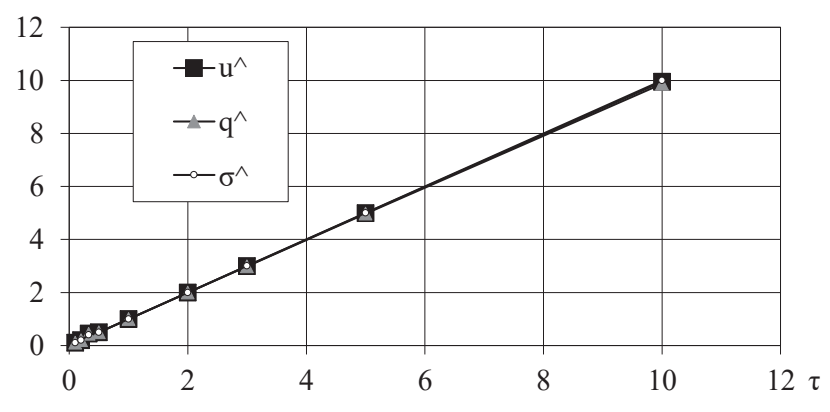

Fig. 15. Dependence of change in the non-dimensional characteristics $\hat{q}, \hat{\sigma}, \hat{u}$ on a degree of variation $\tau_{Q}=\tau_{N}=\tau_{F}=\tau$

\section{Discussion of results from the analysis of trends in the change of the distribution of the SSS components and the contact pressure in the examined objects}

To analyze the SSS in the system of elastic bodies, which are in contact along the aligned surfaces, we have constructed a numerical based on a finite element method. The contact problem is reduced to minimizing the quadratic form, which approximated the functionality of full energy of the examined system on a set of nodal displacements. Within the space of these nodal displacements, the contact constraints are the hyperplanes that pass through the coordinate origin. Given such peculiarities, the problem solution, as shown by Fig. 2, is directly proportional to the current forces. That is, if an unconditional minimum of the quadratic form is reached, it increases directly in proportion to the current forces due to the linearity of the system of equations $\partial I / \partial U_{e}=0, \partial I / \partial U_{g}=0$. If the condition $U_{e}=U_{g}$ is met, that is the case of equality in ratio (7), a different, but the linear equation $\partial I / \partial U_{g}=0$ remains. In both cases, the solution is directly proportional to forces $f$.

Thus, based on the defined pattern of the numerical model of the contact interaction between bodies and the matching surfaces, we have proposed a semi-analytic method for analyzing the contact interaction between structural elements along the aligned surfaces. It implies finding a solution to similar contact problems at the random level of active forces based on the solution at a certain level of these forces by a direct proportional scaling.

The resulting solutions to the test problems confirm the projected dependences of the distribution of the contact pressure, the stresses, and displacements, on the existing forces. Thus, under a misaligned variation of the individual components of the system of existing forces one observes sharp changes in the patterns of the SSS distribution (Fig. 11). If the active forces are directly proportional to a single parameter, the SSS characteristics are directly proportional to this parameter (Fig. 7, 15).

The proposed method has advantages over traditional numerical statements because it sharply limits the required number of solutions to the problems on the contact interaction in a multivariant study involving the varying levels of operating forces.

The defined features in the distribution of the contact pressure and the SSS components (Fig. 4-6, 8-10, 12-14) are important in the analysis of displacement balance in technological systems (the type of «part-machine-tool-assembly», which was examined in this work). For such systems, it becomes possible to linearize the solutions to problems on the displacement of points at assemblies for certain ratios between forces: fixing atop a machine, clamping parts, and cutting. The problem was stated in a general form (without a reference to the number of components from the sets of operating forces); the numerical solutions were considered only for a three-component system of forces. This constraint is to be removed in the further studies. In addition, it is expected to determine the sensitivity of a solution to the problem on the contact interaction when varying the geometric shape of surfaces of the examined bodies.

\section{Conclusions}

1. We have proposed a model of the stressed-strained state taking into consideration the contact interaction between bodies along the aligned surfaces. This model is based on a variational statement. In this case, it takes into consideration the features when there is a zero gap between the bodies in contact. Discretizing the problem by using a finite element method reduces the problem to minimizing the quadratic form under one-sided constraints in the form of linear homogeneous ratios.

2. The discretized model of the contact interaction between bodies along the aligned surfaces makes it possible to predict a directly proportional dependence of the components of the stressed-strained state of these bodies on the level of the current forces provided their components are proportional to a single parameter. Accordingly, at the design stage of estimating the elements of structures, when one needs multi-optional studies into the stressed-strained state taking into consideration their contact interaction, it becomes possible to reduce the amount of computation. The SSS of these bodies is calculated at a certain set of forces. Varying the levels of forces accordingly changes the calculation results, despite the nonlinearity of analysis problems in a general case.

3. We have confirmed, using machine assemblies of the vise type as an example, the dependences of the components of the stressed-strained state on the level of operating forces, predicted on the basis of an analysis of the structure of the mathematical and numerical model of the stressed-strained state. Contact areas remain unchanged when the level of operating forces changes. The magnitudes of the contact pressure and the SSS components grow directly in proportion to the current forces.

\section{References}

1. Johnson, K. L. (1985). Contact Mechanics. Cambridge University Press. doi: https://doi.org/10.1017/cbo9781139171731

2. Rogovyi, A. (2018). Energy performances of the vortex chamber supercharger. Energy, 163, 52-60. doi: https://doi.org/10.1016/ j.energy.2018.08.075

3. Rogovyi, A., Khovanskyy, S. (2017). Application of the similarity theory for vortex chamber superchargers. IOP Conference Series: Materials Science and Engineering, 233, 012011. doi: https://doi.org/10.1088/1757-899x/233/1/012011 
4. Rogovyi, A., Khovanskyy, S., Grechka, I., Pitel, J. (2019). The Wall Erosion in a Vortex Chamber Supercharger Due to Pumping Abrasive Mediums. Advances in Design, Simulation and Manufacturing II, 682-691. doi: https://doi.org/10.1007/ 978-3-030-22365-6_68

5. Gaydamaka, A., Kulik, G., Frantsuzov, V., Hrechka, I., Khovanskyi, S., Rogovyi, A. et. al. (2019). Devising an engineering procedure for calculating the ductility of a roller bearing under a no-central radial load. Eastern-European Journal of Enterprise Technologies, 3 (7 (99)), 6-10. doi: https://doi.org/10.15587/1729-4061.2019.168145

6. Syomin, D., Rogovyi, A. (2012). Features of a Working Process and Characteristics of Irrotational Centrifugal Pumps. Procedia Engineering, 39, 231-237. doi: https://doi.org/10.1016/j.proeng.2012.07.029

7. Panchenko, A., Voloshina, A., Boltyansky, O., Milaeva, I., Grechka, I., Khovanskyy, S. et. al. (2018). Designing the flow-through parts of distribution systems for the PRG series planetary hydraulic motors. Eastern-European Journal of Enterprise Technologies, 3 (1 (93)), 67-77. doi: https://doi.org/10.15587/1729-4061.2018.132504

8. Panchenko, A., Voloshina, A., Kiurchev, S., Titova, O., Onopreychuk, D., Stefanov, V. et. al. (2018). Development of the universal model of mechatronic system with a hydraulic drive. Eastern-European Journal of Enterprise Technologies, 4 (7 (94)), 51-60. doi: https://doi.org/10.15587/1729-4061.2018.139577

9. Panchenko, A., Voloshina, A., Milaeva, I., Panchenko, I., Titova, O. (2018). The Influence of the form Error after Rotor Manufacturing on the Output Characteristics of an Orbital Hydraulic Motor. International Journal of Engineering \& Technology, 7 (4.3), 1. doi: https://doi.org/10.14419/ijet.v7i4.3.19542

10. Voloshina, A., Panchenko, A., Boltynskiy, O., Panchenko, I., Titova, O. (2018). Justification of the Kinematic Diagrams for the Distribution System of a Planetary Hydraulic Motor. International Journal of Engineering \& Technology, 7 (4.3), 6. doi: https://doi.org/10.14419/ijet.v7i4.3.19544

11. Voloshina, A., Panchenko, A., Boltyansky, O., Titova, O. (2019). Improvement of Manufacture Workability for Distribution Systems of Planetary Hydraulic Machines. Advances in Design, Simulation and Manufacturing II, 732-741. doi: https://doi.org/ 10.1007/978-3-030-22365-6_73

12. Martynyak, R. M., Slobodyan, B. S. (2009). Contact of elastic half spaces in the presence of an elliptic gap filled with liquid. Materials Science, 45 (1), 66-71. doi: https://doi.org/10.1007/s11003-009-9156-9

13. Slobodyan, B. S., Lyashenko, B. A., Malanchuk, N. I., Marchuk, V. E., Martynyak, R. M. (2016). Modeling of Contact Interaction of Periodically Textured Bodies with Regard for Frictional Slip. Journal of Mathematical Sciences, 215 (1), 110-120. doi: https:// doi.org/10.1007/s10958-016-2826-x

14. Kravchuk, A. S., Neittaanmäki, P. J. (2007). Variational and Quasi-Variational Inequalities in Mechanics. Springer. doi: https:// doi.org/10.1007/978-1-4020-6377-0

15. Vollebregt, E., Segal, G. (2014). Solving conformal wheel-rail rolling contact problems. Vehicle System Dynamics, 52 (sup1), 455-468. doi: https://doi.org/10.1080/00423114.2014.906634

16. Kalker, J. J. (1977). Variational Principles of Contact Elastostatics. IMA Journal of Applied Mathematics, 20 (2), $199-219$. doi: https://doi.org/10.1093/imamat/20.2.199

17. Papangelo, A., Hoffmann, N., Ciavarella, M. (2017). Load-separation curves for the contact of self-affine rough surfaces. Scientific Reports, 7 (1). doi: https://doi.org/10.1038/s41598-017-07234-4

18. Ciavarella, M. (2015). Adhesive rough contacts near complete contact. International Journal of Mechanical Sciences, 104, 104-111. doi: https://doi.org/10.1016/j.ijmecsci.2015.10.005

19. Ciavarella, M., Joe, J., Papangelo, A., Barber, J. R. (2019). The role of adhesion in contact mechanics. Journal of The Royal Society Interface, 16 (151), 20180738. doi: https://doi.org/10.1098/rsif.2018.0738

20. Li, Q., Popov, V. L. (2018). Adhesive force of flat indenters with brush-structure. Facta Universitatis, Series: Mechanical Engineering, 16 (1), 1. doi: https://doi.org/10.22190/fume1712200051

21. Li, S., Yao, Q., Li, Q., Feng, X.-Q., Gao, H. (2018). Contact stiffness of regularly patterned multi-asperity interfaces. Journal of the Mechanics and Physics of Solids, 111, 277-289. doi: https://doi.org/10.1016/j.jmps.2017.10.019

22. Popov, V. L., Pohrt, R., Li, Q. (2017). Strength of adhesive contacts: Influence of contact geometry and material gradients. Friction, 5 (3), 308-325. doi: https://doi.org/10.1007/s40544-017-0177-3

23. Ciavarella, M. (2017). A very simple estimate of adhesion of hard solids with rough surfaces based on a bearing area model. Meccanica, 53 (1-2), 241-250. doi: https://doi.org/10.1007/s11012-017-0701-6

24. Ciavarella, M. (2017). On Pastewka and Robbins' Criterion for Macroscopic Adhesion of Rough Surfaces. Journal of Tribology, 139 (3). doi: https://doi.org/10.1115/1.4034530

25. Argatov, I., Li, Q., Pohrt, R., Popov, V. L. (2016). Johnson-Kendall-Roberts adhesive contact for a toroidal indenter. Proceedings of the Royal Society A: Mathematical, Physical and Engineering Sciences, 472 (2191), 20160218. doi: https://doi.org/ 10.1098/rspa.2016.0218

26. Ciavarella, M. (2018). An approximate JKR solution for a general contact, including rough contacts. Journal of the Mechanics and Physics of Solids, 114, 209-218. doi: https://doi.org/10.1016/j.jmps.2018.03.005

27. Li, Q., Pohrt, R., Lyashenko, I. A., Popov, V. L. (2019). Boundary element method for nonadhesive and adhesive contacts of a coated elastic half-space. Proceedings of the Institution of Mechanical Engineers, Part J: Journal of Engineering Tribology, 234 (1), 73-83. doi: https://doi.org/10.1177/1350650119854250 
28. Li, Q., Popov, V. L. (2017). Boundary element method for normal non-adhesive and adhesive contacts of power-law graded elastic materials. Computational Mechanics, 61 (3), 319-329. doi: https://doi.org/10.1007/s00466-017-1461-9

29. Rey, V., Anciaux, G., Molinari, J.-F. (2017). Normal adhesive contact on rough surfaces: efficient algorithm for FFT-based BEM resolution. Computational Mechanics, 60 (1), 69-81. doi: https://doi.org/10.1007/s00466-017-1392-5

30. Atroshenko, O., Tkachuk, M. A., Martynenko, O., Tkachuk, M. M., Saverska, M., Hrechka, I., Khovanskyi, S. (2019). The study of multicomponent loading effect on thinwalled structures with bolted connections. Eastern-European Journal of Enterprise Technologies, 1 (7 (97)), 15-25. doi: https://doi.org/10.15587/1729-4061.2019.154378

31. Atroshenko, O., Bondarenko, O., Ustinenko, O., Tkachuk, M., Diomina, N. (2016). A numerical analysis of non-linear contact tasks for the system of plates with a bolted connection and a clearance in the fixture. Eastern-European Journal of Enterprise Technologies, 1 (7 (79)), 24. doi: https://doi.org/10.15587/1729-4061.2016.60087

32. Tkachuk, M. M., Skripchenko, N. B., Tkachuk, M. A. (2016). Solving of problems on contact interaction of rough bodies using model of nonlinear winkler layer. Mekhanika ta mashynobuduvannia, 1, 3-14.

33. Tkachuk, M. (2018). A numerical method for axisymmetric adhesive contact based on Kalker's variational principle. EasternEuropean Journal of Enterprise Technologies, 3 (7 (93)), 34-41. doi: https://doi.org/10.15587/1729-4061.2018.132076

34. Tkachuk, M. M., Skripchenko, N., Tkachuk, M. A., Grabovskiy, A. (2018). Numerical methods for contact analysis of complex-shaped bodies with account for non-linear interface layers. Eastern-European Journal of Enterprise Technologies, 5 (7 (95)), 22-31. doi: https://doi.org/10.15587/1729-4061.2018.143193

35. Tkachuk, M., Bondarenko, M., Grabovskiy, A., Sheychenko, R., Graborov, R., Posohov, V. et. al. (2018). Thinwalled structures: analysis of the stressedstrained state and parameter validation. Eastern-European Journal of Enterprise Technologies, 1 (7 (91)), 18-29. doi: https://doi.org/10.15587/1729-4061.2018.120547

36. Tkachuk, N. A., Kravchenko, S. A., Pylev, V. A., Parsadanov, I. V., Grabovsky, A. V., Veretelnik, O. V. (2019). Discrete and Continual Strengthening of Contacting Structural Elements: Conception, Mathematical and Numerical Modeling. Science \& Technique, 18 (3), 240-247. doi: https://doi.org/10.21122/2227-1031-2019-18-3-240-347

37. Bondarenko, M., Tkachuk, M., Grabovskiy, A., Hrechka, I. (2019). Substantiation of Thin-Walled Structures Parameters Using Nonlinear Models and Method of Response Surface Analysis. International Journal of Engineering Research in Africa, 44, 32-43. doi: https://doi.org/10.4028/www.scientific.net/jera.44.32

38. Washizu, K. (1982). Variational Methods in Elasticity \& Plasticity. Oxford-New York: Pergamon Press, 630.

39. Zienkiewicz, O. C., Taylor, R. L., Zhu, J. Z. (2013). The Finite Element Method: Its Basis and Fundamentals. Butterworth-Heinemann, 756. doi: https://doi.org/10.1016/c2009-0-24909-9

40. Karmanov, V. G. (2004). Matematicheskoe programmirovanie. Moscow: FIZMATLIT, 264.

41. Tkachuk, M. M., Grabovskiy, A., Tkachuk A., M., Hrechka, I., Ishchenko, O., Domina, N. (2019). Investigation of multiple contact interaction of elements of shearing dies. Eastern-European Journal of Enterprise Technologies, 4 (7 (100)), 6-15. doi: https:// doi.org/10.15587/1729-4061.2019.174086

42. Tkachuk, M., Grabovskiy, A., Tkachuk, M. M., Zarubina, A., Saverska, M., Mukhin, D., Kutsenko, S. (2019). Contact interaction of molds elements and project-technological provision of their technical. Bulletin of the National Technical University «KhPI» Series: Engineering and CAD, 1, 58-66. doi: https://doi.org/10.20998/2079-0775.2019.1.07

43. Sea, Zh. (1973). Optimizatsiya. Teoriya i algoritmy. Moscow: Mir, 244.

44. Himmel'blau, D. M. (1975). Prikladnoe nelineynoe programmirovanie. Moscow, 534. 